\title{
X-ray Crystal Structure of the Cytochrome P450 2B4 Active Site Mutant F297A in Complex with Clopidogrel: Insights into Compensatory Rearrangements of the Binding Pocket ${ }^{\dagger}$
}

\author{
Manish B. Shah ${ }^{1,3}$, Hyun-Hee Jang ${ }^{1,3,}{ }^{*}$, Qinghai Zhang ${ }^{2}$, C. David Stout ${ }^{2}$, and James R. \\ Halpert $^{1}$ \\ ${ }^{1}$ Skaggs School of Pharmacy and Pharmaceutical Sciences, University of California, San Diego, \\ La Jolla, CA 92093, United States \\ 2Department of Molecular Biology, The Scripps Research Institute, La Jolla, CA 92037, United \\ States
}

\section{Abstract}

Prior X-ray crystal structures of cytochrome P450 2B4 revealed the pivotal role of rearrangement of the side chains of residues F206 and F297 in the active site in accommodating various inhibitors or substrates. To explore the role of these residues, 2B4 F206A and F297A were created by site-directed mutagenesis and characterized functionally. The structure of F297A with clopidogrel demonstrated the reorientation of the ligand such that the methyl ester group is oriented toward the heme, whereas the thiophene moiety now extends to the additional void in the F297A mutant. Most interestingly, movement of the I helix and several amino acid side chains within the active site was observed in apparent response to the altered binding orientation. Results of flexible docking using the 2B4 wild type or the F297A-virtual mutant positioned either the thiophene or chlorophenyl group closer to heme. However, docking of clopidogrel using the real F297A mutant or a virtual mutant with the I-helix re-positioned oriented clopidogrel preferentially with either the methyl ester or the chlorophenyl group closest to heme. The study provides insight into how the altered active site adapts to accommodate and interact with the substrate in a distinct orientation while maintaining the overall closed protein conformation.

\section{Keywords}

Cytochrome P450; P450 2B4 F297A; Clopidogrel; X-ray Crystal Structure; Ligand Docking

\section{Introduction}

The cytochrome P450 superfamily catalyzes the monooxygenation of a variety of endogenous and exogenous compounds and provides a critical pathway for the metabolic clearance of drugs and toxicants in human [1]. P450-mediated catalysis generally involves

\footnotetext{
$\dagger$ This research was supported by NIH grants ES003619 to J.R.H and GM098538 to Q.Z. Atomic coordinates have been deposited at the Protein Data Bank (accession code 4H1N).

(C) 2013 Published by Elsevier Inc.

*Corresponding Author: hyj006@ucsd.edu; Tel: 858-822-7804; Fax: 858-246-0089.

${ }^{3}$ These authors contributed equally to this work.

Publisher's Disclaimer: This is a PDF file of an unedited manuscript that has been accepted for publication. As a service to our customers we are providing this early version of the manuscript. The manuscript will undergo copyediting, typesetting, and review of the resulting proof before it is published in its final citable form. Please note that during the production process errors may be discovered which could affect the content, and all legal disclaimers that apply to the journal pertain.
} 
the incorporation of a single oxygen atom into an organic ligand and often occurs with remarkable stereo- and regiospecificity [2]. Cytochromes P450 of the 2B subfamily are inducible by barbiturates and are very versatile catalysts with a broad range of substrates including drugs, environmental pollutants, and steroids [3]. In addition, P450 2B enzymes exhibit a relatively low degree of catalytic preservation across mammalian species and have provided an excellent model system for structure-function analysis [4-6]. Rabbit P450 2B4, in particular, was the first microsomal P450 to be purified and is one of the bestcharacterized xenobiotic metabolizing P450s.

Previous structural and solution studies of N-terminal truncated and modified P450 2B4 ${ }^{\mathrm{a}}$ have revealed a large degree of structural flexibility in the presence of various ligands. Studies using isothermal titration calorimetry are also consistent with high plasticity of the protein in the presence of various imidazole inhibitors [7]. Early X-ray crystal structures of 2B4 in complex with 4-(4-chlorophenyl)imidazole (4-CPI) or 1-(4-chlorophenyl)imidazole (1-CPI) revealed a closed enzyme conformation $[8,9]$. This conformation resembles those of subsequent complexes with the anti-platelet drugs ticlopidine or clopidogrel, a covalent complex with tBPA, and a closed ligand-free structure [10-12]. Considerably more open conformations have been observed with the larger ligand bifonazole and in the absence of ligand [13]. The open tBPA-modified CYP2B4 structure also resembles this open conformation. However, the 1-PBI complex of 2B4 adopts a conformation that is intermediate to the closed and bifonazole-bound structures [14].

In addition to overall conformational flexibility, our recently determined crystal structures of 2B4 and 2B6 have provided a detailed look at side chain rearrangements needed to accommodate the respective ligands within the active site. In particular, the closed forms of 2B4 and 2B6 exhibit a reorientation of Phe206 and Phe297 [8-10, 15]. In the 2B4 complexes with 1-CPI and clopidogrel and the closed tBPA-modified P450 2B4 structure, Phe206 enters the active site, while Phe297 rotates out. In the 4-CPI complexes of 2B6 and 2B4, the closed 2B4 ligand-free, and ticlopidine complex of 2B4, these two residues exchange positions, so that Phe206 points out and Phe297 points in [16].

Recent results with other mammalian P450 enzymes have shown that the flexibility and malleability of P450 1A2, 2A6, 2C9 and 3A4 enzymes assessed by molecular dynamics (MD) simulation, UV/VIS and resonance Raman spectroscopy correlates with their substrate specificity [17]. Consistent with the structures, this work showed that P450 1A2 and 2A6 have small and rigid active sites and show high substrate specificity. In contrast, P450 3A4 displays the highest substrate promiscuity, corresponding to the most malleable active site, and P450 2C9 is intermediate in terms of both its substrate specificity and malleability. In addition, X-ray structures of active site mutants of mammalian P450 enzymes have also provided insight as to how these enzymes can adapt to bind their respective ligands [18]. More recently, functional and structural analysis of wild type (WT) P450 2A13, 2A6, 2E1 and 2A6 mutants have allowed direct comparisons that reveal how individual residues lining the active sites interact differently with the heme iron-bound inhibitor pilocarpine [19].

Previous studies in our laboratory performed prior to the availability of X-ray crystal structures have identified key residues responsible for substrate specificity of P450 2B forms including rat 2B1 [20, 21], rabbit 2B4 and 2B5 [5, 22, 23], dog 2B11 [6, 24] and human 2B6 [25] by analysis of allelic variants with altered specificities and by site-directed mutagenesis.

aThe initial P450 2B4 X-ray crystal structure was solved using a truncated and modified protein containing the wild type His 226. Because of the formation of a dimer involving coordination of $\mathrm{His}^{226}$ of each monomer with the heme iron of the other monomer, subsequent biochemical and crystallography work utilized the mutant H226Y. In this manuscript, 2B4 or 2B4 WT will refer to P450 2B4dH (H226Y) with N-terminal truncations, C-terminal His tag and H226Y mutation unless otherwise stated [8]. 
In those studies, homology models based on bacterial P450s or later P450 2C5 were used as templates to understand mutagenesis results. The basic premise was that the only structural change in the active site was the substituted residue. However, in light of the demonstrated flexibility of P450 2B enzymes, it became important to explore by X-ray crystallography how a ligand would bind in the active site of a mutant $2 \mathrm{~B} 4$. For this purpose we chose F297A, a mutant that retains high binding affinity for the drug clopidogrel, a ligand that is not anchored in the active site by a coordinate bond to the heme iron [10], in contrast to most other ligand complexes we have studied. Despite an overall protein fold highly similar to that of the 2B4-clopidogrel complex, the experimentally determined structure of $\mathrm{P} 450$ 2B4 F297A was significantly different from the above WT complex in regards to the active site topology and ligand orientation. The findings demonstrate the compensatory effect required to facilitate drug binding in an altered active site.

\section{Materials and Methods}

\section{Materials}

Clopidogrel and $\beta$-NADPH were purchased from Sigma-Aldrich (St. Louis, MO). 7 Hydroxy-4-(trifluoromethyl)coumarin, and 7-ethoxy-4-(trifluoromethyl)coumarin (7-EFC) were purchased from Invitrogen (Carlsbad, CA). 5-Cyclo-hexylpentyl- $\beta$-D-maltoside (Cymal-5) was obtained from Anatrace (Maumee $\mathrm{OH}$ ). The $50 \mathrm{kDa}$ molecular mass protein cutoff Amicon Ultra filtration devices were from Millipore (Billerica, MA). Nickelnitrilotriacetic acid affinity resin was from Qiagen (Valencia, CA), and Macroprep CM cation exchange resin was obtained from Bio-Rad Laboratories (Hercules, CA). The QuikChange XL site-directed mutagenesis kit was obtained from Stratagene (La Jolla, CA). The Index HT crystal screen was obtained from Hampton Research (Aliso Viejo, CA). 3a, $7 a, 12 a$-Tris[ $(\beta$-D-maltopyranosyl) ethyloxy]cholane (232-chol) is a custom made facial amphiphile [26]. TOPP3 cells were from Stratagene (La Jolla, CA). Recombinant NADPH cytochrome $\mathrm{P} 450$ reductase (CPR) and cytochrome $b_{5}$ from rat liver were prepared as described previously [27]. All other chemicals and supplies used were from standard sources. The structural figures were created using PyMOL [28].

\section{Site-directed Mutagenesis}

The mutants F206A and F297A were generated using pKK2B4dH(H226Y) plasmid [8] as a template by polymerase chain reaction with Stratagene's QuikChange XL site-directed mutagenesis kit. The forward primers $5^{\prime}$ TTGTTCTTCCAGTCCGCCTCCCTCATCAGCTCC- $3^{\prime}$ and $5^{\prime}$ TGCTCTCGCTCTTCGCCGCCGGCACC-3' were used for the Phe to Ala conversion at positions 206 and 297, respectively. The changes in the nucleotide bases are underlined. The site-specific mutations and absence of any spurious mutations were confirmed by DNA sequencing at Retrogen Inc (San Diego, CA, USA).

\section{Protein Expression and Purification}

P450 2B4 F206A and F297A mutants were expressed and purified in a similar manner as described for 2B4 [10]. Briefly, protein expression was induced in Escherichia coli that had been transformed with the vector pKK2B 4 by the addition of isopropyl $\beta$-D-1thiogalactopyranoside and $\delta$-aminolevulinic acid supplemented Terrific broth medium $\left(A_{600} \sim 0.7\right.$ at $37^{\circ} \mathrm{C}$ ) in the presence of tetracycline and ampicillin. Protein expression continued for $68-72 \mathrm{~h}$ at $30^{\circ} \mathrm{C}$, and the cells were harvested by centrifugation $(4,000 \mathrm{~g})$ and lysed. After ultracentrifugation in the presence of high salt and the detergent Cymal-5 (Anatrace), the supernatant was purified using $\mathrm{Ni}^{2+}$-NTA metal affinity chromatography which was washed with buffer containing $100 \mathrm{mM}$ potassium phosphate $\left(\mathrm{pH} 7.4\right.$ at $\left.4^{\circ} \mathrm{C}\right)$, $100 \mathrm{mM} \mathrm{NaCl}, 20 \%$ (v/v) glycerol, $10 \mathrm{mM}$ 2-mercaptoethanol (BME), $0.5 \mathrm{mM}$ PMSF, 4.8 
$\mathrm{mM}$ Cymal-5, and $1 \mathrm{mM}$ histidine. The protein was eluted using $40 \mathrm{mM}$ histidine in the same buffer described above. Pooled P450-containing fractions were diluted 10-fold in buffer containing $5 \mathrm{mM}$ potassium phosphate $\left(\mathrm{pH} 7.4\right.$ at $4^{\circ} \mathrm{C}$ ), $20 \%$ (v/v) glycerol, $1 \mathrm{mM}$ EDTA, $0.2 \mathrm{mM}$ dithiothreitol (DTT), $0.5 \mathrm{mM}$ PMSF, and $4.8 \mathrm{mM}$ Cymal-5, before being loaded onto a Macroprep CM cation exchange column. The column was washed using 5 $\mathrm{mM}$ potassium phosphate ( $\mathrm{pH} 7.4$ at $4^{\circ} \mathrm{C}$ ), $20 \mathrm{mM} \mathrm{NaCl}, 20 \%$ (v/v) glycerol, $1 \mathrm{mM}$ EDTA, and $0.2 \mathrm{mM}$ DTT, and the protein was eluted with high-salt buffer containing $50 \mathrm{mM}$ potassium phosphate (pH 7.4 at $4^{\circ} \mathrm{C}$ ), $500 \mathrm{mM} \mathrm{NaCl}, 20 \%$ (v/v) glycerol, $1 \mathrm{mM}$ EDTA, and $0.2 \mathrm{mM}$ DTT. Protein fractions with the highest $A_{41} A_{280}$ ratios were pooled, and the P450 content was measured from the reduced CO-difference spectrum [29].

\section{Enzymatic Assays}

7-EFC $O$-deethylation was measured in a final volume of $100 \mu 1$ as described previously [30]. The reaction mixture contained 10 pmol P450, 40 pmol cytochrome $\mathrm{P} 450$ reductase, and 20 pmol cytochrome $b_{5}$ in $50 \mathrm{mM}$ HEPES, $15 \mathrm{mM} \mathrm{MgCl}_{2}, 0.1 \mathrm{mM}$ EDTA (pH 7.6). The samples were incubated at $37^{\circ} \mathrm{C}$ for 5 minutes before the addition of $1 \mathrm{mM} \mathrm{NADPH}$ to initiate the reaction. After 5 minutes the reaction was terminated by the addition of $20 \%$ trichloroacetic acid, and then $950 \mu \mathrm{l}$ of Tris- $\mathrm{HCl}$ buffer ( $\mathrm{pH}$ 9.0) was added to the reaction mixture $(50 \mu \mathrm{l})$. The metabolites were quantitated by measuring 7-HFC at $\lambda_{\mathrm{ex}} 410 \mathrm{~nm}$ and $\lambda_{\text {em }}$ of $510 \mathrm{~nm}$, respectively. Steady state kinetic parameters were determined by regression analysis using GraphPad Prism (GraphPad Software, San Diego, CA). The $K_{\mathrm{m}}$ and $k_{\mathrm{cat}}$ values were calculated using the Michaelis-Menten equation.

\section{Spectral Binding Titration}

Ligand binding to $2 \mathrm{~B} 4$ mutants was determined at $25^{\circ} \mathrm{C}$ by titrating $1 \mu \mathrm{M}$ enzyme in buffer containing $50 \mathrm{mM}$ potassium phosphate (pH 7.4), $500 \mathrm{mM} \mathrm{NaCl}, 1 \mathrm{mM}$ EDTA, $0.2 \mathrm{mM}$ DTT, and $500 \mathrm{mM}$ sucrose. Final $\mathrm{CH}_{3} \mathrm{OH}$ concentrations were $\varsigma$ s\% (v/v). Difference spectra $(350 \sim 500 \mathrm{~nm})$ were recorded after each addition of clopidogrel (dissolved in methanol) in a $1.0 \mathrm{ml}$ total volume on an MC2000-2 multichannel CCD rapid scanning spectrometer (Ocean Optics, Dunedin, FL, USA) using a semi-micro quartz cell with a stirring compartment $(10 \times 4 \mathrm{~mm}$ light path) from Hellma $\mathrm{GmbH}$ (Mülheim, Germany) as described previously [7]. The spectral dissociation constant $\left(K_{\mathrm{S}}\right)$ were obtained by fitting the data to the equation for "tight binding" $2 \Delta \mathrm{A}=2 \Delta \mathrm{A}=\left(\mathrm{A}_{\max } /\left[\mathrm{E}_{0}\right]\right)-\left[\mathrm{K}_{\mathrm{D}}+\left[\mathrm{I}_{0}\right]+\left[\mathrm{E}_{0}\right]+\left(\mathrm{K}_{\mathrm{D}}\right.\right.$ $\left.\left.+\left[\mathrm{I}_{0}\right]+\left[\mathrm{E}_{0}\right]\right)^{2}-\left(4\left[\mathrm{E}_{0}\right]\left[\mathrm{I}_{0}\right]\right)^{1 / 2}\right]$ for high affinity ligand using SpectraLab software [31].

\section{Enzyme Inhibition}

$I C_{50}$ values were determined as described previously [32] using 7-EFC O-deethylation measured in a final reaction volume of $100 \mu \mathrm{l}$ at clopidogrel concentrations of $0.1-5 \mu \mathrm{M}$ with a substrate concentration of $50 \mu \mathrm{M}$. The activities were plotted against inhibitor concentration in SigmaPlot (Systat Software, Inc., Point Richmond, CA). The data were fit to a four-parameter logistic function by linear regression analysis to derive the inhibitory concentration $50 \%\left(I C_{50}\right)$ value.

\section{Crystallization and Data collection}

Pooled protein was diluted to $18 \mu \mathrm{M}$ in $50 \mathrm{mM}$ potassium phosphate ( $\mathrm{pH} 7.4$ ), $500 \mathrm{mM}$ sucrose, $500 \mathrm{mM} \mathrm{NaCl}, 1 \mathrm{mM}$ EDTA, and $0.2 \mathrm{mM}$ DTT and then mixed with a 10 -fold excess of clopidogrel. This solution was incubated overnight at $4^{\circ} \mathrm{C}$ and then concentrated until the protein concentration was $550 \mu \mathrm{M}$. The concentrated protein-inhibitor complex was supplemented with $4.8 \mathrm{mM}$ Cymal-5, $1 \mathrm{mM}$ clopidogrel, and $0.028 \%(\mathrm{w} / \mathrm{v}) 232$-cholate. The sitting-drop vapor diffusion method was used to screen crystallization conditions using the Hampton Research Index screen. Crystals of 2B4 F297A with clopidogrel were obtained at 
$18^{\circ} \mathrm{C}$ after incubating the protein in a $1: 1$ ratio with the precipitant containing $25 \%(\mathrm{w} / \mathrm{v})$ PEG 3350, 0.1 M Bis-Tris (pH 6.5), and 0.2 M ammonium sulfate. Crystals were soaked in a solution containing $20 \%(\mathrm{v} / \mathrm{v})$ sucrose as cryoprotectant and were flash frozen in liquid nitrogen. Crystallographic data were collected remotely at Stanford Synchrotron Radiation Lightsource (SSRL) beam line 7-1 [33] using $1^{\circ}$ oscillations over 240 frames and $20 \mathrm{~s}$ exposures using Quantum 315 CCD detector at $100 \mathrm{~K}$ temperature. Crystals of 2B4 F297A diffracted to $2.9 \AA$ resolution and data were integrated using iMOSFLM [34] and scaled using SCALA in CCP4 [35].

\section{Structure Determination and Refinement}

The structure of 2B4 F297A in complex with clopidogrel was determined using the coordinates of the closed ligand-free 2B4 structure (PDB entry 3MVR) as a starting model in the molecular replacement program Phaser [36] in CCP4. Matthews coefficient analysis determined the presence of one molecule in the asymmetric unit with $63.5 \%$ solvent content, and the space group was $\mathrm{P}_{1} 21$ as determined by Phaser. A rigid body and restrained refinement was performed in REFMAC using the output model from Phaser, and the model was built in COOT [37] using $F_{o}-F_{c}$ and $2 F_{o}-F_{c}$ electron density maps contoured at $3 \sigma$ and $1 \sigma$, respectively. PRODRG server was used to make the library description for clopidogrel [38]. Manual model building followed by iterative rounds of refinement was continued until the R-factor and R-free stopped improving. The model was validated by MOLPROBITY [39], which ranked the structure in the $100^{\text {th }}$ percentile among structures of comparable resolution, yielding no Ramachandran outliers or outlier bond lengths or angles. A total of 19 molecules of water and three molecules of CYMAL-5 detergent (two with partial occupancy) were found to be present in the final model of the crystal structure, which contained native protein residues from 28 to 491 and a residue 492 as the first histidine of the C-terminal His-tag. The C-D loop residues 135-138, found previously to interact with cytochrome $\mathrm{P} 450$ reductase [40], and residues 473-474 were disordered in the final model. Coordinates and structure factors were deposited in the Protein Data Bank (PDB entry $4 \mathrm{H} 1 \mathrm{~N})$, and the refinement statistics for the above structure are summarized in Table 1.

\section{Ligand Docking and Active Site Volume Calculation}

AutoDock Vina version 1.1.1 [41] was used to dock clopidogrel into the 2B4 F297A complex (PDB entry 4H1N), 2B4 WT complex (PDB entry 3ME6) [10] and 2B4 F297A virtual mutant (2B4 VM) created using the coordinates of 2B4 WT complex with clopidogrel. The mutation at residue F297 to alanine was made in COOT in the 2B4clopidogrel structure by deleting the side chain of Phe and renaming as alanine, and the file was subsequently named as 2B4 VM. Ligand molecules that include clopidogrel, Cymal-5, sulfate ion and water molecules were removed from the PDB file, and docking was run using a rigid receptor molecule. Gasteiger charges were used for small molecules, and charges on heme were based on previously reported values using a separate script [42]. The docking experiment included 20 events with a grid size of $70 \AA \times 70 \AA \times 70 \AA$ centered on the heme iron. Moreover, a flexible receptor file was generated using AutoDockTools4 after selecting residues located near the active site, which were allowed to move freely. These residues included I101, V104, F108, I114, F115, F202, S205, F206, I209, L238, S294, F296, F297, A298, G299, T300, E301, T302, T303, L362, I363, V367, V477, whereas all the other residues of the protein were maintained in the rigid receptor file.

The active site cavity volume was calculated using Voidoo [43], using a probe occupied volume with a probe radius of $1.4 \AA$. The clopidogrel, water, sulfate ion and Cymal-5 molecules were deleted from the coordinates of 2B4 F297A, and the region above the active site heme iron was chosen as the cavity search. 


\section{Results}

\section{Catalytic Activity of P450 2B4 Mutants}

In order to determine the effect of these mutations on catalytic activity, the steady-state kinetic properties of 2B4 WT and the F206A and F297A mutants were measured with the representative substrate 7-EFC (Table 2). F206A showed 6-fold lower $k_{\text {cat }}$, and 1.8-fold higher $K_{\mathrm{m}}$ for 7-EFC, which results in a catalytic efficiency for this substrate 11-fold lower than 2B4 WT. Compared with WT, the $k_{\text {cat }}$ and $K_{\mathrm{m}}$ of F297A were decreased by 1.2 -fold and 1.6-fold, whereas the catalytic efficiency was essentially unaltered.

\section{Spectral Binding and Enzyme Inhibition}

The binding affinities of clopidogrel for 2B4 WT, F206A, and F297A were determined by monitoring ligand induced heme absorbance changes. Clopidogrel induced a type I difference spectra with a peak at $\sim 388 \mathrm{~nm}$ and a trough at $\sim 420 \mathrm{~nm}$. F297A exhibited a similar $\Delta A_{\max }$ value as $2 \mathrm{~B} 4 \mathrm{WT}$, whereas the $\Delta A_{\max }$ for $\mathrm{F} 206 \mathrm{~A}$ was 2.8 -fold lower than WT enzyme. Compared with 2B4 WT, F206A and F297A showed approximately 2-fold higher $K_{\mathrm{S}}$ values with clopidogrel (Table 3). $I C_{50}$ values were also determined using 7-EFC at a concentration of $50 \mu \mathrm{M}$ and drug concentrations of $0-5 \mu \mathrm{M}$. F297A exhibited a 3-fold lower $I C_{50}$ value for clopidogrel $(0.4 \mu \mathrm{M}$ vs. $1.2 \mu \mathrm{M})$ than $2 \mathrm{~B} 4 \mathrm{WT}$, whereas the lower activity of F206A precluded accurate $I C_{50}$ determination.

\section{Structure of P450 2B4 (F297A) in Complex with Clopidogrel}

The crystal structure of 2B4 F297A in complex with clopidogrel was solved at $2.9 \AA$ resolution. The difference electron density for the ligand was clearly defined near the heme prior to inclusion of the drug in the model (Figure 1A). Moreover, the difference electron density of the phenylalanine side chain at position 297 was not observed based on the $2 F o$ $F C$ and $F o-F c$ maps, which clearly showed the presence of alanine at residue 297 . The complete structure of 2B4 F297A is shown in Figure 1B. A total of 18 residues were found to be located within a $5 \AA$ radius of clopidogrel in the complex, which form the predominantly hydrophobic active site (Figure 1C). These include residues I101, V104 and F108 on the B' helix; I114 and F115 on the B' - C loop; F202, S205, F206 and I209 on the F helix; L238 on the G helix; A297, A298, E301, T302 on the I helix; I363 and V367 on the K-K' loop; and V477 and G478 near the $\beta_{4}$ sheet region. Of these, residues F202, S205 and L238 were not observed within a $5 \AA$ A radius of the ligand in previously solved structures of 2B4. [44]. The absence of the F297 side chain now allows the thiophene ring to rotate $90^{\circ}$ compared with the 2B4 WT complex, filling the void region and placing the ligand in close proximity to residues F202, S205 and L238. F202 forms a base of the hydrophobic pocket where a Cymal-5 molecule is bound in this as well as in several other previously solved structures. Residues F206 and F297 have been shown to rearrange to accommodate various ligands. However, mutation at F297 to alanine did not affect the orientation of the F206 side chain when compared with the 2B4 WT-clopidogrel complex and several other structures $[10,16]$. The overall structure is very similar to the WT-clopidogrel complex (rmsd $\sim 0.39$ $\AA$ ) and is in a canonical closed conformation. The chlorophenyl ring of clopidogrel reorients towards the $\beta 4$ loop and the K-K' loop in the F297A complex, as opposed to the WT complex where this ring was located near heme. The methyl ester group of clopidogrel is located near the heme in the new structure, with the closest atom at a distance of $5.5 \AA$ from the heme iron.

In addition to the altered orientation of the bound clopidogrel there are some noteworthy rearrangements of some residues that line the active site. Particularly, the chlorophenyl group of clopidogrel is sequestered near residue V477 where the side chain is flipped upside down by about $180^{\circ}$ in order to avoid clash with the ligand. This movement was not 
observed in the WT complex, in which the chlorophenyl was located near the heme. Such movement and the smaller side chain of F297A contribute to a larger active site volume of $552 \AA^{3}$ compared with the 2B4 WT clopidogrel complex $\left(417 \AA^{3}\right)$ [11] as shown in Figure 2A. In addition, there is a kink in the I helix mainly from residues F296 to T303, which protrude by up to $1.4 \AA$ into the F297A active site (Figure 2B). A similar kink was previously observed in the 2B4-ticlopidine complex and the 1-CPI complex, where the main chain carbonyls of residues 296-299 lose their hydrogen bonding partners of the main chain amides from residues 300-303 [9, 10].

\section{Ligand Docking}

To correlate the orientation of clopidogrel in silico with that observed in the crystal structures, molecular docking studies were performed, starting with the 2B4 WT complex. In general, a range of binding energies is observed in the docking experiments for a particular ligand, some of which are very similar. This makes it important to consider the possibility of any pose being the most favorable, unless a significant difference in energy is observed. In our analysis, we considered all poses within $\sim 0.5 \mathrm{kcal} / \mathrm{mol}$ of the lowest energy one. In rigid docking the lowest energy pose $(-7.4 \mathrm{kcal} / \mathrm{mol})$ had the thiophene group oriented towards the heme. The two subsequent lowest energy poses had either the chlorophenyl moiety closer to heme consistent with the X-ray crystal structure or the methyl ester moiety facing the heme (energies of -7.3 and $-7.2 \mathrm{kcal} / \mathrm{mol}$, respectively). However, rigid docking into the virtual mutant 2B4 VM created from the 2B4 WT complex showed either the chlorophenyl or the methyl ester group down in the first 10 lowest energy poses $(-8.5$ to $-7.8 \mathrm{kcal} / \mathrm{mol})$. The thiophene group was not observed near the heme in any of these poses and was always located near A297 in the region above the I helix. When clopidogrel was docked into a model based on the 2B4 F297A complex, the methyl ester group oriented towards the heme in the lowest energy pose $(-8.5 \mathrm{kcal} / \mathrm{mol})$, and the chlorophenyl group was located near the $\beta_{4}$ loop region, which is consistent with the X-ray crystal structure. However, the next two poses had either the thiophene $(-8.4 \mathrm{kcal} / \mathrm{mol})$ or the chlorophenyl moiety $(-8.3 \mathrm{kcal} / \mathrm{mol})$ closer to heme, suggesting that clopidogrel could orient with any of these groups toward the heme.

Flexible docking yielded intriguing results due to more freedom for the ligand to orient within the active site. The 2B4 WT complex had mostly the chlorophenyl or thiophene ( -9.0 $\mathrm{kcal} / \mathrm{mol}$ ) of clopidogrel down as the lowest energy poses (Figure 3A and 3B). A similar trend was also observed with the 2B4 VM, where either the chlorophenyl or thiophene moiety $(-8.7 \mathrm{kcal} / \mathrm{mol})$ was closer to heme in the lowest energy and most populous cluster of poses (Figure 3C and 3D). However, in the 2B4 F297A mutant structure, the first 5 poses (energies ranging from -8.6 to $-8.1 \mathrm{kcal} / \mathrm{mol}$ ) had either chlorophenyl or methyl ester down orientation (Figure 4A and 4B). The thiophene down orientation was not observed in these poses most likely due to the movement of the I-helix residues 296 to 303. Furthermore, to investigate the role of these residues, the I-helix (residues from 284-332) of the 2B4-F297A structure was superimposed onto the $2 \mathrm{~B} 4-\mathrm{VM}$ structure in COOT, and the resulting structure was used for docking studies. Interestingly, the thiophene down orientation was not observed until the $7^{\text {th }}$ pose with the lowest energy $(-8.1 \mathrm{kcal} / \mathrm{mol})$. The $1^{\text {st }}$ and $2^{\text {nd }}$ pose had either the chlorophenyl group or the methyl ester group closer to heme with energies of -8.9 and $-8.8 \mathrm{kcal} / \mathrm{mol}$, respectively (Figure $4 \mathrm{C}$ and $4 \mathrm{D}$ ). In the flexible docking the protrusion of the I helix into the active site appears to preclude the bulky thiophene group from being close to the heme.

\section{Discussion}

In the current study the consequences of substituting F206 or F297 in 2B4 with alanine was investigated using site-directed mutagenesis, X-ray crystallography, and functional and 
computational analysis. As observed previously, F206A was considerably less active than 2B4 toward 7-EFC [9]. The results are consistent with our prior mutagenesis studies of P450 2B1, which showed that F206L exhibited a markedly altered steroid metabolite profile along with decreased rates of alkoxycoumarin $O$-dealkylation. [45, 46]. Furthermore, F297A and F297W in P450 2B1 demonstrated a significant reduction in testosterone hydroxylation, while F297A and F297I exhibited altered metabolite profiles with 7-butoxycoumarin [47]. Only F297W displayed a 7-butoxycoumarin metabolism profile similar to WT P450 2B1 suggesting the importance of an aromatic ring at this position. Furthermore, the P450cam residue analogous to 2B4 F297 was found to directly affect substrate binding [48]. These functional analyses of substitutions at F206 and F297 residues show the pivotal role they play in determining catalytic activity of $2 \mathrm{~B} 4$.

Because 2B4 F297A largely retained the high affinity of the WT enzyme for clopidogrel along with high expression in $E$. coli, the mutant was studied using X-ray crystallographic analysis. The structure of the F297A clopidogrel complex of 2B4 revealed important rearrangements of residues in the vicinity of the active site compared with the WT complex. The position of clopidogrel itself in the new structure now allows several phenylalanine residues located near the active site to re-orient. In particular, residue F203 close to the active site rotates by more than $90^{\circ}$ towards residue F202, which lines the active site. In the absence of F297 in the new structure, F202 forms the lid of the closed active site and is now backed by another phenylalanine residue 203 , as if to compensate for the effect of one phenylalanine mutation. F202 adopts a slightly different conformation in the new structure, where the phenyl ring is facing the thiophene group of clopidogrel, which is located near the void created by replacing F297 with alanine (Figure 5A).

Interestingly, analysis of the current and prior structures reveals that the in and out movement of F297 within the active site is correlated with the binding of Cymal-5 in a peripheral pocket behind the I helix, which is mainly lined by hydrophobic residues. As observed in 2B4-ticlopidine [10], 2B6-4CPI [49], 2B4 ligand free [12] and 2B4-amlodipine [50] structures, a molecule of Cymal-5 was found located near F202, which forms a base of the largely hydrophobic pocket with F203 in similar orientation as seen in the new F297A clopidogrel complex. Residue F297 in these structures rotate by $90^{\circ}$ away from F202. However, in the 2B4-clopidogrel, 2B6-4BP and 2B6-4NBP [15], 2B4-1CPI [9] and 2B4tBPA [11] set of structures, F202 is stacked against F297 in a slightly different conformation, and the cyclohexane group of Cymal-5 detergent does not occupy the peripheral pocket. In the new 2B4 F297A structure, F202 is aligned in a similar fashion as in 2B4-ticlopidine and the other set of structures that exhibit the binding of Cymal-5 molecule in this hydrophobic cavity. Such differences are shown in Figure 5B with representative structures of 2B4 F297A-clopidogrel, 2B4-ticlopidine, 2B4-amlodipine, 2B4-clopidogrel and 2B4-1CPI complexes. Additionally, residue F203 forms a wall of the pocket in those structures that contain Cymal-5, whereas in the 2B4 WT-clopidogrel and 2B4-1CPI complexes, residue 203 rotates out of this pocket not occupied by the detergent molecule. All these rearrangements of the cluster of phenylalanine residues located close or within the active site, either on F helix or I helix may play an important role in interplay between the peripheral pocket and the active site in ligand binding.

The structural alignment of 2B4 WT and F297A in complex with clopidogrel demonstrated a shift of part of the I helix toward the active site that could not have been anticipated based solely on a single mutation. Accordingly, rigid docking of clopidogrel using the structure of the actual F297A mutant as a template, but not a virtual mutant, predicted the same orientation as that observed in the structure. However, manual repositioning of the I helix in the WT complex, creating the virtual mutant, and performing flexible docking yielded results that were consistent with those observed with the F297A structure. Specifically, in 
both cases orientation of the thiophene group of clopidogrel toward the heme was energetically less favorable than other orientations with the chlorophenyl or methyl ester groups close to the heme. Overall, the docking studies further explain the differences observed in clopidogrel orientation in two very similar structures with an amino acid substitution and movement of the I helix within the active site.

Comparison of the structure of 2B4 F297A with that of other P450 enzymes with mutations at the corresponding location yields some new insights. F297 in 2B4 maps to I300 in P450 $2 \mathrm{~A} 6$ and $\mathrm{F} 300$ in P450 2A13. Recent analysis of 2A13, 2A6 and the active site mutant 2A6 I208S/I300F/G301A/S369G in complex with pilocarpine illustrated the effect of substitution at position 300 [19]. The overall conformation of the P450 2A structures in complex with pilocarpine was similar to that of the 2B4 WT and 2B4 F297A clopidogrel complexes. Interestingly, the secondary structural elements in the active sites of the P450 2A6 WT, 2A6 mutant, and 2A13 structures are essentially superimposable, in contrast to the pronounced movement of the I helix toward the active site in the 2B4 F297A structure. This is consistent with the flexibility of 2B compared with 2A enzymes. In the P450 2A6 I300F mutant the ethyl group of the furan ring of pilocarpine is located towards residue 300 and away from the conserved F118 and L370, in contrast to P450 2A6 WT. This observation is consistent with the pilocarpine orientation in the P450 2A13 structure. The side chain difference at residue 300 was found to be one of the most important contributing features in the active sites of P450 2A6 and P450 2A13. This was further demonstrated by a separate study where the residue at position 300 was shown to be critical in the metabolism of phenacetin, a substrate of P450 2A13 but not of 2A6 [51]. The increased phenacetin activity in the P450 2A6 I208S/I300F/G301A/G369S mutant resulted in part from the release of a steric clash with the $\mathrm{I} 300$ side chain in the active site as evident from the structural and docking analysis [51]. In addition, the phenylalanine substitution at this location was also shown to be crucial for binding of several other ligands of P450 2A enzymes [52]. Despite the clear importance of the side chain at this position in P450 2A and 2B enzymes, it is important to note that F297 is invariant among the rat, rabbit, dog, and human liver P450 2B enzymes and thus cannot account for species differences in function.

In conclusion, the current structure presents the first snapshot of an active site mutant of a P450 2B enzyme. The study emphasizes the structural and functional implications of residue substitutions within the active site upon binding a clinical drug. The results obtained enabled us to extract more detail on the orientation of ligand itself and several other residues using different computational methods, which would be difficult to interpret based on the available WT structure in complex with clopidogrel. Finally, the structure presented here allowed the comparison with three different P450 2A complexes, one involving the mutation at a similar location on the I helix that demonstrated altered metabolism and ligand orientation compared to that of the cognate structure. The biochemical and biophysical analysis of additional active site residue substitutions will be required in addition to the mutations of the phenylalanine residues in the vicinity of the active site, which will further aid in understanding substrate recognition and specificity of P450 2B enzymes.

\section{Acknowledgments}

We thank the staff at the Stanford Synchrotron Radiation Lightsource, operated by Stanford University on behalf of the United States Department of Energy, Office of Basic Energy Sciences for assistance with data collection. The Stanford Synchrotron Radiation Lightsource is supported by the National Institute of Health, the National Center for Research Resources, the Biomedical Technology Program, and the United States Department of Energy of Biological and Environmental Research. 


\section{References}

1. Johnson EF, Stout CD. Biochem Biophys Res Commun. 2005; 338:331-336. [PubMed: 16157296]

2. Guengerich FP. Chem Res Toxicol. 2001; 14:611-650. [PubMed: 11409933]

3. Domanski TL, Halpert JR. Curr Drug Metab. 2001; 2:117-137. [PubMed: 11469721]

4. Zhao Y, Halpert JR. Biochim Biophys Acta. 2007; 1770:402-412. [PubMed: 16935426]

5. Kedzie KM, Philpot RM, Halpert JR. Arch Biochem Biophys. 1991; 291:176-186. [PubMed: 1929429]

6. Kedzie KM, Grimm SW, Chen F, Halpert JR. Biochim Biophys Acta. 1993; 1164:124-132. [PubMed: 8329443]

7. Muralidhara BK, Negi S, Chin CC, Braun W, Halpert JR. J Biol Chem. 2006; 281:8051-8061. [PubMed: 16439365]

8. Scott EE, White MA, He YA, Johnson EF, Stout CD, Halpert JR. J Biol Chem. 2004; 279:2729427301. [PubMed: 15100217]

9. Zhao Y, Sun L, Muralidhara BK, Kumar S, White MA, Stout CD, Halpert JR. Biochemistry. 2007; 46:11559-11567. [PubMed: 17887776]

10. Gay SC, Roberts AG, Maekawa K, Talakad JC, Hong WX, Zhang Q, Stout CD, Halpert JR. Biochemistry. 2010; 49:8709-8720. [PubMed: 20815363]

11. Gay SC, Zhang H, Wilderman PR, Roberts AG, Liu T, Li S, Lin HL, Zhang Q, Woods VL, Stout CD, Hollenberg PF, Halpert JR. Biochemistry. 2011:4903-4911. [PubMed: 21510666]

12. Wilderman PR, Shah MB, Liu T, Li S, Hsu S, Roberts AG, Goodlett DR, Zhang Q, Woods VL Jr, Stout CD, Halpert JR. J Biol Chem. 2010; 285:38602-38611. [PubMed: 20880847]

13. Zhao Y, White MA, Muralidhara BK, Sun L, Halpert JR, Stout CD. J Biol Chem. 2006; 281:59735981. [PubMed: 16373351]

14. Gay SC, Sun L, Maekawa K, Halpert JR, Stout CD. Biochemistry. 2009; 48:4762-4771. [PubMed: 19397311]

15. Shah MB, Pascual J, Zhang Q, Stout CD, Halpert JR. Mol Pharmacol. 2011; 80:1047-1055. [PubMed: 21875942]

16. Halpert JR. Drug Metab Dispos. 2011; 39:1113-1121. [PubMed: 21502194]

17. Hendrychova T, Anzenbacherova E, Hudecek J, Skopalik J, Lange R, Hildebrandt P, Otyepka M, Anzenbacher P. Biochim Biophys Acta. 2010; 1814:58-68. [PubMed: 20656072]

18. Sansen S, Hsu MH, Stout CD, Johnson EF. Arch Biochem Biophys. 2007; 464:197-206. [PubMed: 17540336]

19. DeVore NM, Meneely KM, Bart AG, Stephens ES, Battaile KP, Scott EE. FEBS J. 2012; 279:1621-1631. [PubMed: 22051186]

20. He YA, Balfour CA, Kedzie KM, Halpert JR. Biochemistry. 1992; 31:9220-9226. [PubMed: 1390709]

21. Halpert JR, He YA. J Biol Chem. 1993; 268:4453-4457. [PubMed: 8440727]

22. Ryan R, Grimm SW, Kedzie KM, Halpert JR, Philpot RM. Arch Biochem Biophys. 1993; 304:454-463. [PubMed: 8346920]

23. Szklarz GD, He YQ, Kedzie KM, Halpert JR, Burnett VL. Arch Biochem Biophys. 1996; 327:308-318. [PubMed: 8619620]

24. Graves PE, Elhag GA, Ciaccio PJ, Bourque DP, Halpert JR. Arch Biochem Biophys. 1990; 281:106-115. [PubMed: 2116765]

25. Spatzenegger M, Liu H, Wang Q, Debarber A, Koop DR, Halpert JR. J Pharmacol Exp Ther. 2003; 304:477-487. [PubMed: 12490624]

26. Zhang Q, Ma X, Ward A, Hong WX, Jaakola VP, Stevens RC, Finn MG, Chang G. Angew Chem Int Ed Engl. 2007; 46:7023-7025. [PubMed: 17691085]

27. Harlow GR, He YA, Halpert JR. Biochim Biophys Acta. 1997; 1338:259-266. [PubMed: 9128144]

28. DeLano, WL., editor. MacPyMOL. DeLano Scientific; Palo Alto, CA: 2002.

29. Omura T, Sato R. J Biol Chem. 1964; 239:2370-2378. [PubMed: 14209971] 
30. Scott EE, Spatzenegger M, Halpert JR. Arch Biochem Biophys. 2001; 395:57-68. [PubMed: 11673866]

31. Davydov DR, Deprez E, Hoa GH, Knyushko TV, Kuznetsova GP, Koen YM, Archakov AI. Arch Biochem Biophys. 1995; 320:330-344. [PubMed: 7625841]

32. Hernandez CE, Kumar S, Liu H, Halpert JR. Arch Biochem Biophys. 2006; 455:61-67. [PubMed: 17027909]

33. Soltis SM, Cohen AE, Deacon A, Eriksson T, Gonzalez A, McPhillips S, Chui H, Dunten P, Hollenbeck M, Mathews I, Miller M, Moorhead P, Phizackerley RP, Smith C, Song J, van dem Bedem H, Ellis P, Kuhn P, McPhillips T, Sauter N, Sharp K, Tsyba I, Wolf G. Acta Crystallogr D Biol Crystallogr. 2008; 64:1210-1221. [PubMed: 19018097]

34. Leslie AGW. Acta Crystallogr Sect D-Biol Crystallogr. 1999; 55:1696-1702. [PubMed: 10531519]

35. Bailey S. Acta Crystallogr Sect D-Biol Crystallogr. 1994; 50:760-763. [PubMed: 15299374]

36. McCoy AJ, Grosse-Kunstleve RW, Adams PD, Winn MD, Storoni LC, Read RJ. J Appl Crystallogr. 2007; 40:658-674. [PubMed: 19461840]

37. Emsley P, Cowtan K. Acta Crystallogr D Biol Crystallogr. 2004; 60:2126-2132. [PubMed: 15572765]

38. Schuttelkopf AW, van Aalten DM. Acta Crystallogr D Biol Crystallogr. 2004; 60:1355-1363. [PubMed: 15272157]

39. Davis IW, Murray LW, Richardson JS, Richardson DC. Nucleic Acids Res. 2004; 32:W615-619. [PubMed: 15215462]

40. Bumpus NN, Hollenberg PF. J Inorg Biochem. 2010; 104:485-488. [PubMed: 20096935]

41. Trott O, Olson AJ. J Comput Chem. 2010; 31:455-461. [PubMed: 19499576]

42. Helms V, Wade RC. Biophys J. 1995; 69:810-824. [PubMed: 8519982]

43. Kleywegt GJ, Jones TA. Acta Crystallogr D Biol Crystallogr. 1994; 50:178-185. [PubMed: 15299456]

44. Wilderman PR, Halpert JR. Curr Drug Metab. 2012; 13:167-176. [PubMed: 22208531]

45. Luo Z, He YA, Halpert JR. Arch Biochem Biophys. 1994; 309:52-57. [PubMed: 8117113]

46. Kobayashi Y, Fang X, Szklarz GD, Halpert JR. Biochemistry. 1998; 37:6679-6688. [PubMed: 9578551]

47. Domanski TL, He YQ, Scott EE, Wang Q, Halpert JR. Arch Biochem Biophys. 2001; 394:21-28. [PubMed: 11566023]

48. Mueller, EJ.; Loida, PJ.; Sligar, SJ. Plenum Press; New York: 1995. p. 83-142.

49. Gay SC, Shah MB, Talakad JC, Maekawa K, Roberts AG, Wilderman PR, Sun L, Yang JY, Huelga SC, Hong WX, Zhang Q, Stout CD, Halpert JR. Mol Pharmacol. 2010; 77:529-538. [PubMed: 20061448]

50. Shah MB, Wilderman PR, Pascual J, Zhang Q, Stout CD, Halpert JR. Biochemistry. 2012; 51:7225-7238. [PubMed: 22909231]

51. DeVore NM, Smith BD, Wang JL, Lushington GH, Scott EE. Drug Metab Dispos. 2009; 37:1319_ 1327. [PubMed: 19251817]

52. DeVore NM, Smith BD, Urban MJ, Scott EE. Drug Metab Dispos. 2008; 36:2582-2590. [PubMed: 18779312] 


\section{Highlights}

- The crystal structure of a P450 2B4 F297A complex with clopidogrel was determined.

- The orientation of clopidogrel in the active site differed from the wild type.

- Movement of the I helix and other active site residues was also observed in F297A.

- A virtual F297A with the I helix repositioned is a reasonable template for docking.

- The orientation of F202 correlates with detergent binding in a peripheral pocket. 


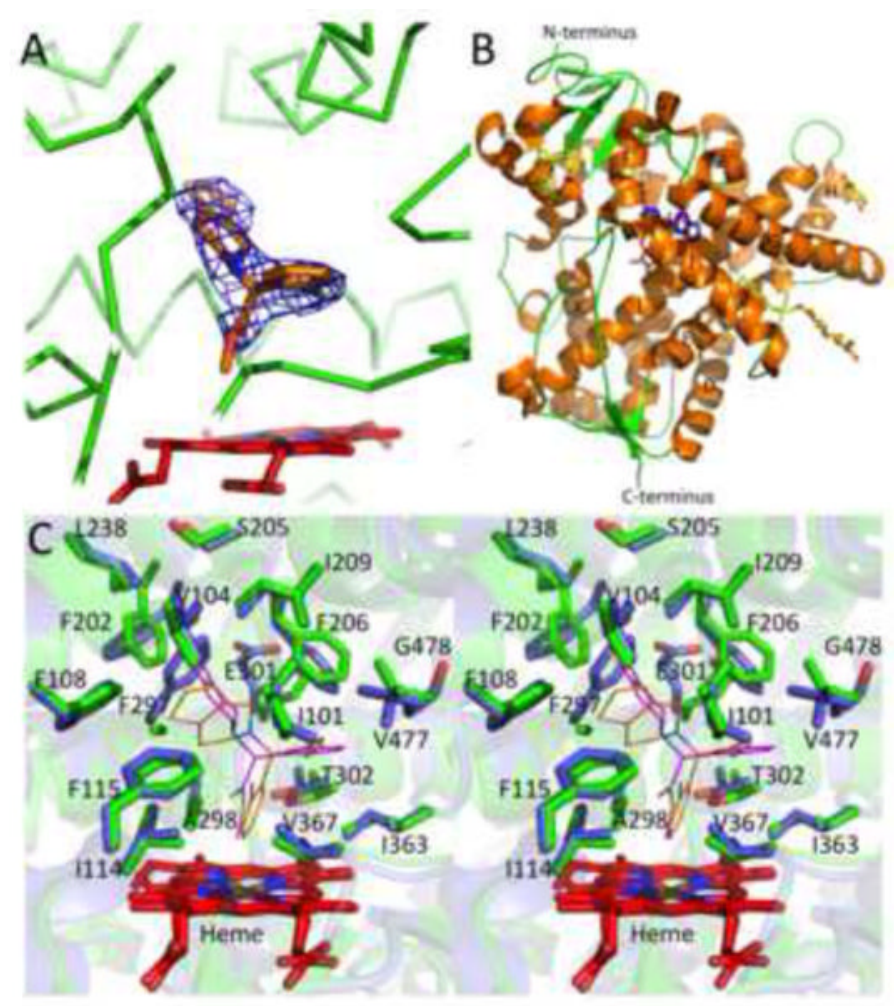

Figure 1.

Omit maps, structure of 2B4 F297A, and overlay of 2B4 F297A with 2B4 WT structure showing residues within $5 \AA$ from the ligand. A. An unbiased $F_{O}-F_{C}$ electron density omit map determined before inclusion of the ligand in the 2B4 F297A complex contoured at $3 \sigma$ corresponding to clopidogrel above heme. B. Crystal structure of 2B4 F297A clopidogrel complex. $a$-helices are shown in orange, and $\beta$-sheets and loops are shown in green. Three Cymal-5 molecules (two partially occupied molecules) and clopidogrel are shown in yellow and blue sticks, respectively. C. Superimposed structures of 2B4 F297A (green) and 2B4 WT (blue) complexes of clopidogrel in stereo view representing active site residues within 5 $\AA$ from the ligand (magenta lines) in 2B4 F297A. The corresponding residue in the 2B4 WT structure in complex with clopidogrel (orange sticks) is also shown. 


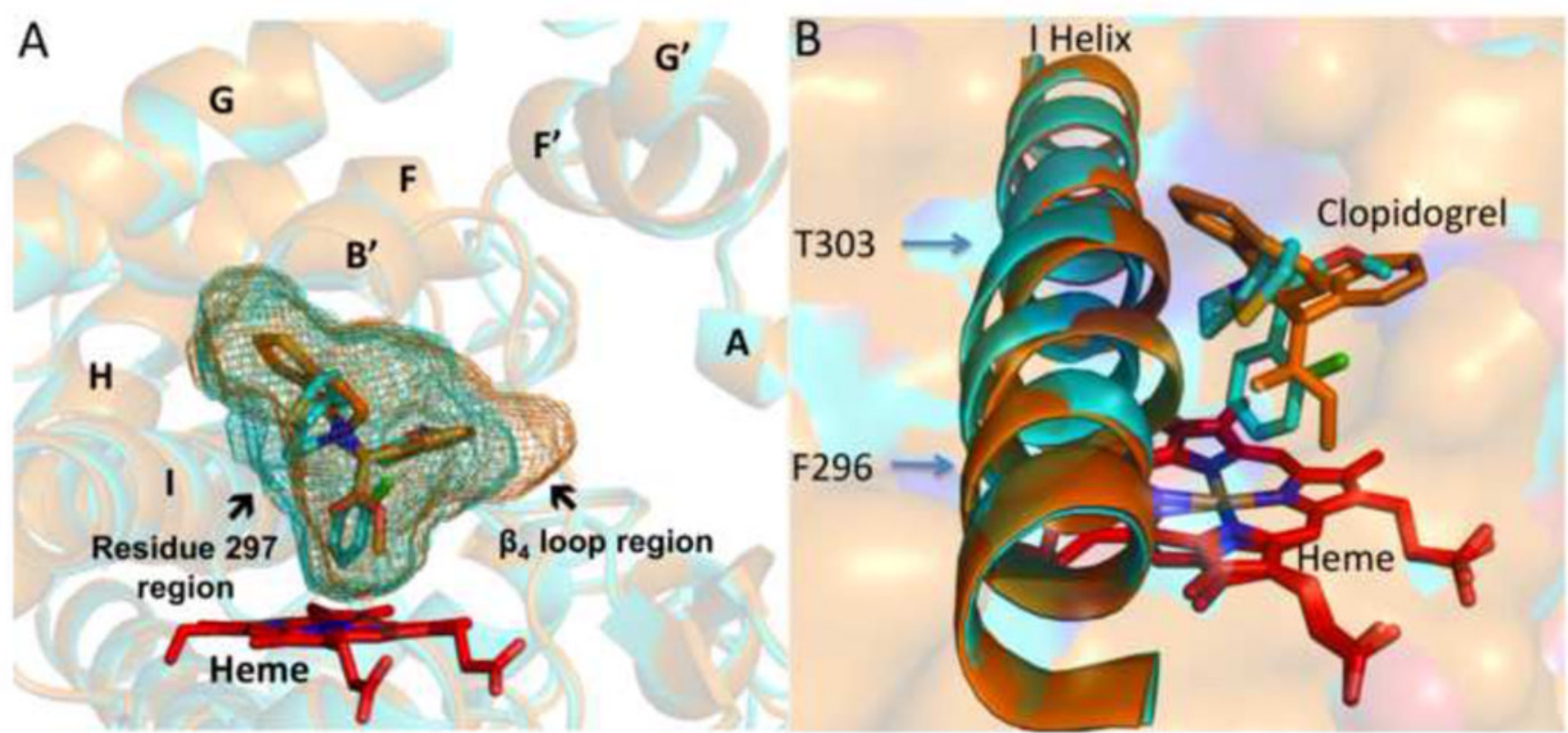

Figure 2.

A. Overlay of cavity volume and the structures of 2B4 F297A (orange) and 2B4 WT (cyan) structures with respective clopidogrel molecule shown in sticks. The cavity volume of the F297A and WT clopidogrel complexes of 2B4 is shown in orange and cyan mesh, respectively. B. Overlay of 2B4 F297A (orange) and 2B4 WT (cyan) structures shown with the I helix in ribbon representation. The residues from F296 to T303 kink into the active site in close vicinity of clopidogrel shown in sticks. 


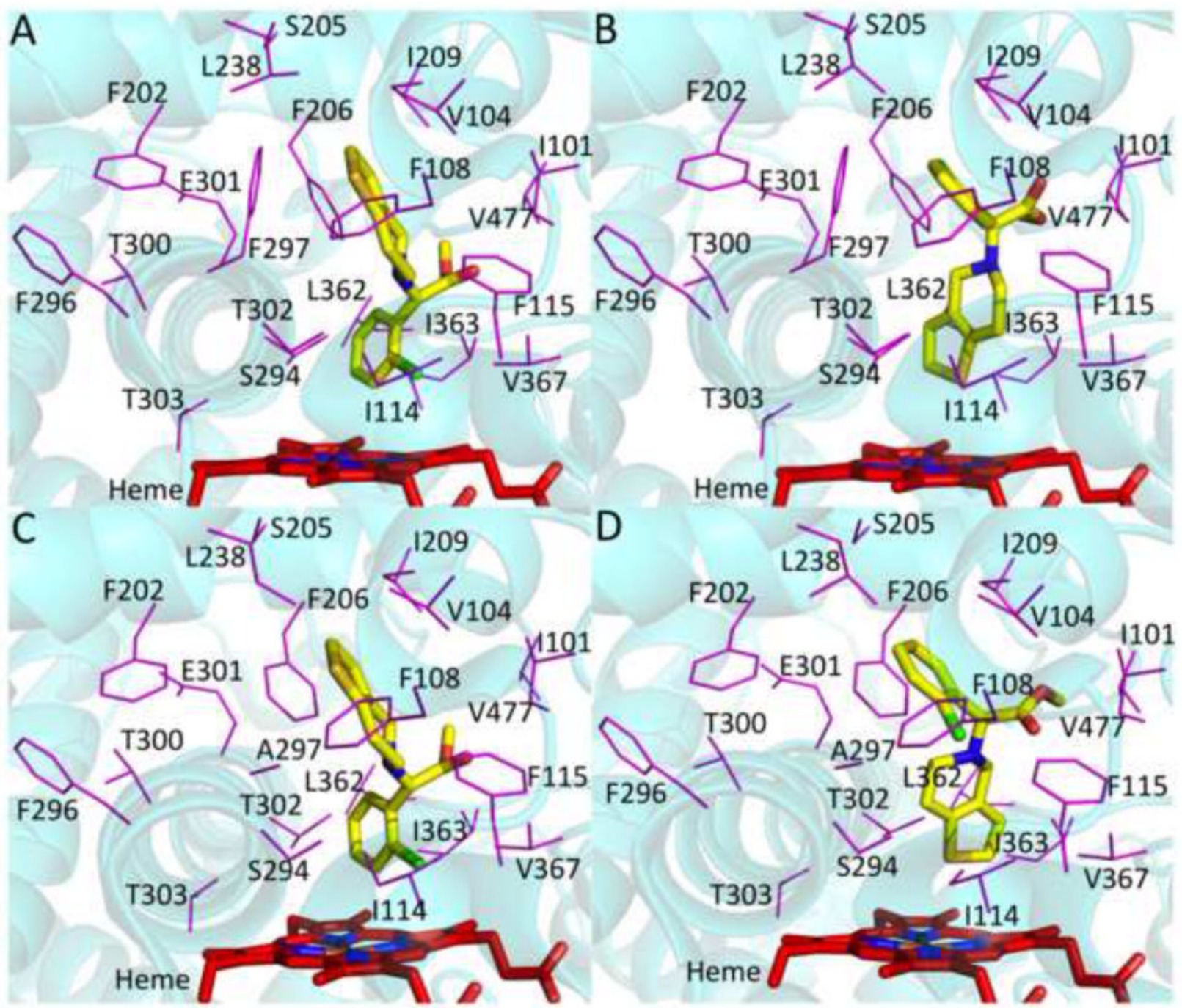

Figure 3.

Flexible docking of clopidogrel into the 2B4 WT and 2B4 VM structures using flexible residues. A. The lowest energy pose with the chlorophenyl moiety of clopidogrel pointing toward the heme in the 2B4 WT docking analysis. B. The pose with the thiophene group of clopidogrel near the heme in the 2B4 WT structure. $\mathrm{C}$ and D. The 2B4 VM docking that shows either chlorophenyl or thiophene down orientation as the preferred poses. 


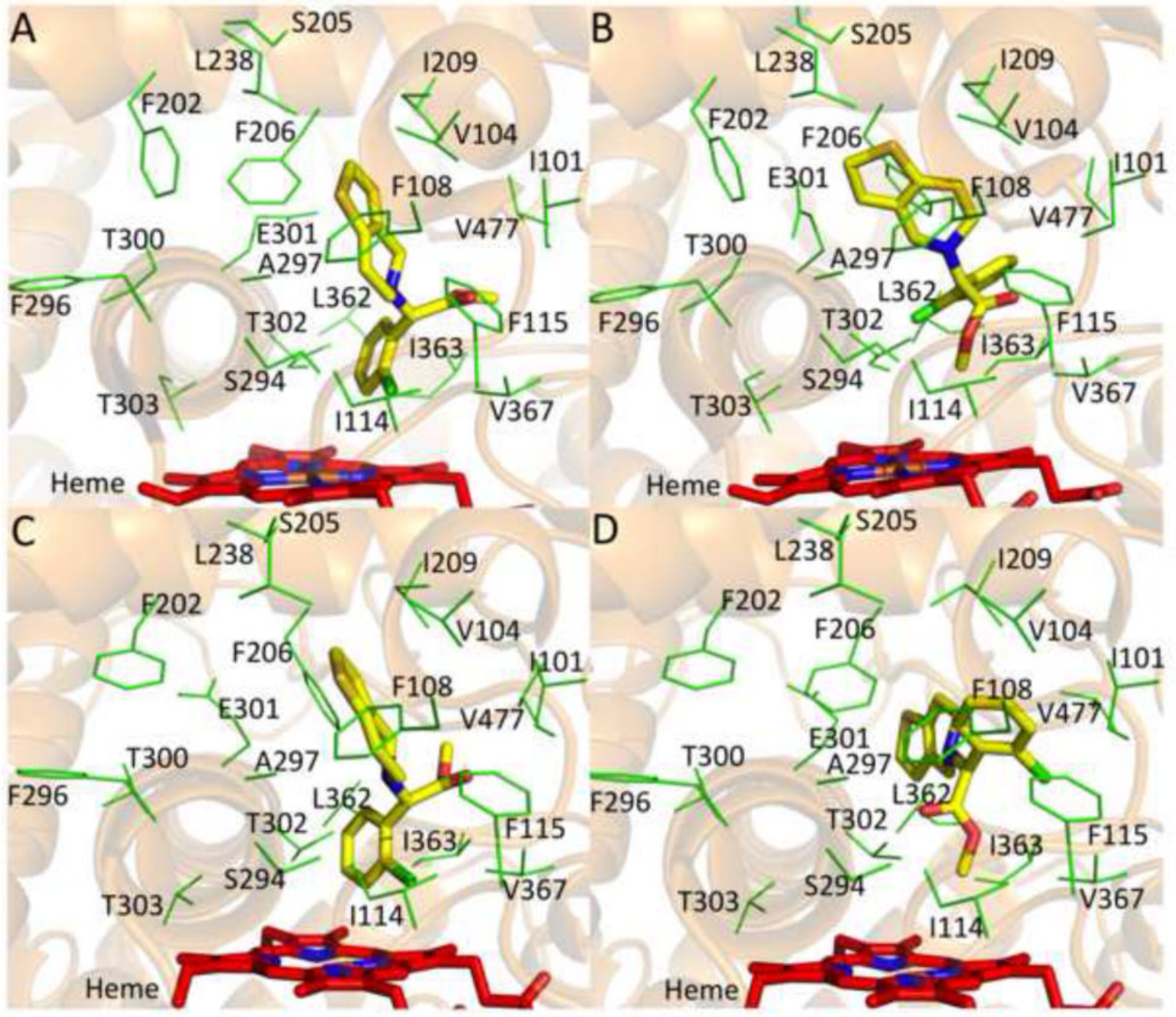

Figure 4.

Flexible docking of clopidogrel into the 2B4 F297A and I helix repositioned 2B4 VM structure showing the most populous cluster of poses. A and B. The poses with lowest energy depicting either the chlorophenyl or methyl ester group of clopidogrel near the heme in the top 5 outcomes with 2B4 F297A. C and D. The chlorophenyl or methyl ester down orientation was consistently observed in at least the first 5 poses, mainly as a result of the shifting of I helix into the active site. 


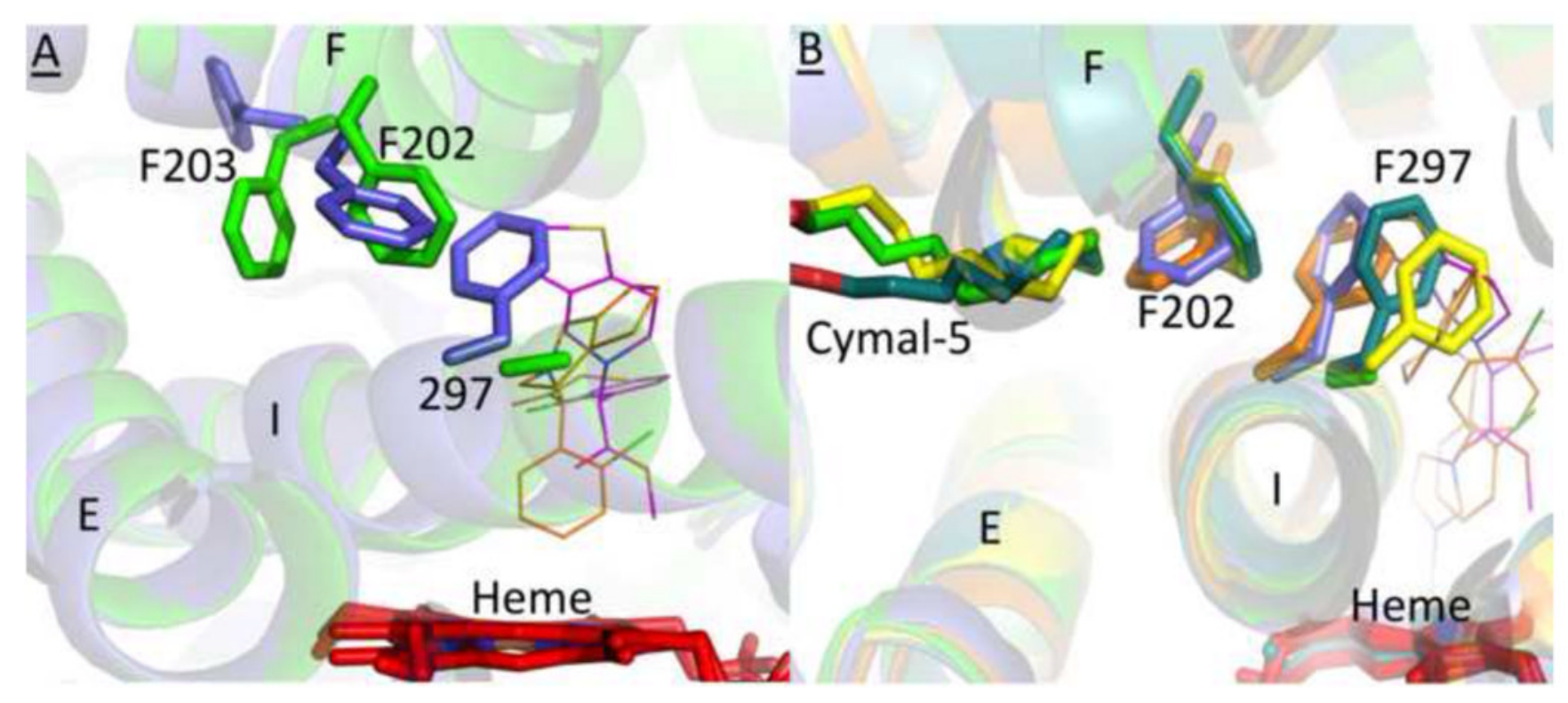

Figure 5.

A. Overlay of 2B4 WT (blue) and 2B4 F297A (green) clopidogrel complexes showing residues F202 and F203 in the vicinity of residue 297. The F202 side chain in 2B4 F297A reorients near the void region of F297 and the thiophene group of clopidogrel. The F203 side chain rotates by $90^{\circ}$ toward F202 compared with 2B4 WT. Clopidogrel is depicted as lines in magenta and orange for 2B4 F297A and 2B4 WT structures, respectively. B. Overlay of 2B4 structures in complex with various ligands. In the 2B4 F297A clopidogrel (green), 2B4 ticlopidine (yellow) and 2B4 amlodipine complexes (dark cyan), F297 rotates by $90^{\circ}$ in the active site and creates space for F202 to reorient, which allows Cymal-5 to bind in the peripheral pocket. In contrast, in the 2B4 WT clopidogrel (blue) and 1CPI (orange) complexes, F202 protrudes into the peripheral pocket, and Cymal-5 is not observed in these structures. 
Table 1

Data collection and refinement statistics of P450 2B4 F297A in complex with clopidogrel. Values for the highest resolution shell are shown in parentheses.

\begin{tabular}{|c|c|}
\hline Crystal space group & $\mathbf{P 3} \mathbf{1}_{1} \mathbf{1}$ \\
\hline \multicolumn{2}{|l|}{ Crystal unit cell parameters } \\
\hline$a=b$ & $91.38 \AA$ \\
\hline $\mathrm{c}$ & $152.28 \AA$ \\
\hline$a=\beta$ & $90^{\circ}$ \\
\hline$\gamma$ & $120^{\circ}$ \\
\hline Molecules per asymmetric unit & 1 \\
\hline \multicolumn{2}{|l|}{ Data Collection } \\
\hline Beam line & SSRL 7-1 \\
\hline Wavelength $(\AA)$ & 0.97 \\
\hline Resolution range ( $\mathrm{A})$ & $39.57-2.9(3.05-2.9)$ \\
\hline Completeness (\%) & $99.1(94.2)$ \\
\hline Redundancy & $13.3(9.3)$ \\
\hline $\mathrm{R}_{\text {merge }}$ & $0.068(0.74)$ \\
\hline $\mathrm{I} / \sigma$ & $10(1.1)$ \\
\hline No. of unique reflections & 16,796 \\
\hline \multicolumn{2}{|l|}{ Refinement } \\
\hline R-factor & $23.3 \%$ \\
\hline R-free & $28.6 \%$ \\
\hline \multicolumn{2}{|l|}{ RMS deviations } \\
\hline Bond lengths $(\AA)$ & 0.007 \\
\hline Bond angles $\left(^{\circ}\right)$ & 1.02 \\
\hline \multicolumn{2}{|l|}{ No. of atoms. } \\
\hline Protein & 3610 \\
\hline Heme & 43 \\
\hline Clopidogrel & 21 \\
\hline Waters & 19 \\
\hline Cymal-5 & 57 \\
\hline Sulfate & 5 \\
\hline \multicolumn{2}{|l|}{ Molprobity - Ramachandran Plot } \\
\hline Preferred $(\%)$ & 95.6 \\
\hline Allowed (\%) & 4.4 \\
\hline
\end{tabular}




\section{Table 2}

Steady state kinetics of P450 2B4 mutants.

\begin{tabular}{cccc}
\hline & \multicolumn{3}{c}{ 7-EFC } \\
\cline { 2 - 4 } Protein & $\boldsymbol{k}_{\text {cat }}\left(\mathbf{m i n}^{-1}\right)$ & $\boldsymbol{K}_{\mathbf{m}}(\mu \mathrm{M})$ & $\boldsymbol{k}_{\text {cat }} / \boldsymbol{K}_{\mathbf{m}}$ \\
\hline 2B4dH & $6.8 \pm 0.4$ & $31.9 \pm 5.5$ & 0.21 \\
F206A & $1.1 \pm 0.1$ & $58.5 \pm 5.4$ & 0.02 \\
F297A & $5.4 \pm 0.2$ & $20.4 \pm 3.1$ & 0.26 \\
\hline
\end{tabular}

- Standard errors ( \pm ) for fit to the Michaelis-Menten equation.

- The result is the representative of at least two independent determinations. 


\section{Table 3}

Interaction of clopidogrel with P450 2B4 mutants assessed by spectral binding and inhibition.

\begin{tabular}{cccc}
\hline Protein & $\boldsymbol{\Delta \boldsymbol { A } _ { \text { max } } ( \mathbf { O U } \boldsymbol { \mu \mathbf { M } ^ { - 1 } } )}$ & $\boldsymbol{K}_{\mathbf{s}}(\boldsymbol{\mu M})$ & $\boldsymbol{I C}_{\boldsymbol{5 0}}(\boldsymbol{\mu M})$ \\
\hline 2B4dH & $0.28 \pm 0.003$ & $0.4 \pm 0.05$ & $1.2 \pm 0.52$ \\
F206A & $0.10 \pm 0.001$ & $0.8 \pm 0.05$ & $\mathrm{ND}$ \\
F297A & $0.27 \pm 0.003$ & $0.9 \pm 0.04$ & $0.4 \pm 0.03$ \\
\hline
\end{tabular}

- Standard errors ( \pm ) for fit to the respective equations are shown. Results are representative of at least two independent determinations. OU stands for optical units. ND, not detectable.

- All data treatment and fitting of the titration curves were performed with our SpectraLab software or using SigmaPlot (Systat Software, Inc., Point Richmond, CA). 